\title{
Identifying an Oxygenation Index Threshold for Increased Mortality in Acute Respiratory Failure
}

\author{
Brandon G Hammond MD MBA, Pamela Garcia-Filion PhD MPH, Paul Kang MPH, \\ Mounica Y Rao, Brigham C Willis MD, and Heidi J Dalton MD
}

\begin{abstract}
BACKGROUND: The objective of this work was to examine current oxygenation index (OI) data and outcomes using electronic medical record data to identify a specific OI value associated with mortality. METHODS: This study was a retrospective electronic medical record data review from the pediatric ICU of Phoenix Children's Hospital, with data mining for variables to calculate OIs on subjects age 1 month to 20 y mechanically ventilated $>24 \mathrm{~h}$, excluding those with known intracardiac shunts or cyanotic heart disease. Age, length of hospital stay, duration of mechanical ventilation, and outcomes were also assessed. The Wilcoxon signed-rank test was used to compare continuous variables, receiver operating characteristic analysis was used in determining discriminant ability, and logistic regression was conducted to determine the odds ratio (OR) for risk of death with increasing OI. RESULTS: OI was calculated on 65 subjects, of whom 6 died $(9 \%)$. The median maximum OI was 10 for all subjects, 17 for non-survivors, and 8 for survivors $(P=.14$ via Wilcoxon rank-sum test). ORs indicated a 2.4-fold increase in the odds of death $(P=.09,95 \%$ CI $0.9-6.6)$ for each increasing point in maximum OI. Mean OI OR revealed a 1.9-fold increase in the odds of death $(P=.25,95 \%$ CI 0.6-5.9). Receiver operating characteristic analysis indicated a higher discriminate ability for maximum OI (area under the curve $=0.68$ ) than mean $\mathrm{OI}$ (area under the curve $=0.58$ ). OI cut-points for mortality were established. Mortality was unchanged until maximum OI $>17$, for which mortality nearly tripled at a value of $18 \%$ versus 6-7\% for range 0-17. CONCLUSIONS: Limitations exist in obtaining serial OI values from current electronic medical records. Serial assessment of OI values may allow creation of alert values for increased mortality risk. Consideration of escalation of therapies for respiratory failure, such as highfrequency ventilation, inhaled nitric oxide, or extracorporeal membrane oxygenation may be warranted at lower OIs than historically reported. Key words: oxygenation index (OI); pediatric ARDS; respiratory severity stratification; outcome measures; practice guidelines; electronic medical records. [Respir Care 2017;62(10):1249-1254. @ 2017 Daedalus Enterprises]
\end{abstract}

\section{Introduction}

Acute respiratory failure is a common cause for admission into pediatric ICUs. Pediatric ARDS is the most se-

Dr Hammond, Dr Garcia-Filion, and Dr Dalton are affiliated with the Division of Critical Care Medicine, and Dr Willis is affiliated with the Division of Cardiovascular Intensive Care Medicine, Phoenix Children's Hospital, Phoenix, Arizona. Dr Hammond, Mr Kang, Ms Rao, Dr Willis, and Dr Dalton are affiliated with the University of Arizona College of Medicine, Phoenix, Arizona.

Dr Dalton has disclosed relationships with Maquet, ThermoFisher, rEVO Biologics, and Innovative ECMO Concepts. The other authors have disclosed no conflicts of interest.

Dr Hammond presented a version of this work in poster sessions at the 31st Annual Children's National Medical Center Symposium, "ECMO and the vere form of a wide spectrum of pathologic processes designated as acute lung injury. ${ }^{1}$ Despite advanced treatments, such as mechanical ventilation, nitric oxide, surfactant delivery, and extracorporeal membrane oxygenation (ECMO), mortality remains high in patients with severe pulmonary disease. ${ }^{2}$ Observational and epidemiologic studies

\footnotetext{
Advanced Therapies for Respiratory Failure," held February 22-26, 2015, in Keystone, Colorado, and at the Pediatric Academic Societies Annual Meeting, held April 25-28, 2015, in San Diego, California.

Correspondence: Brandon Hammond MD MBA, Combined Pediatrics and Anesthesiology Residency Program, University of North Carolina, N2198, CB7010, UNC Hospitals, Chapel Hill, NC 27599-7010. E-mail: Brandon_Hammond@med.unc.edu.
}

DOI: $10.4187 /$ respcare. 05092 
have found mortality rates in pediatric ARDS ranging from 10 to $50 \%$ from 1992 to $2013 .{ }^{3}$

Advances in understanding the pathophysiology of respiratory failure have led to technological improvements in ECMO and other rescue therapy delivery. With these

See the Related Editorial on Page 1375

improvements, previously excluded patient groups with comorbidities and underlying complex chronic problems are receiving rescue therapies, such as ECMO, with increasing frequency. ${ }^{4}$ Well-validated prognostic criteria for implementation of rescue therapies are lacking. Respiratory failure severity calculations, which have been suggested for ECMO candidacy, include the alveolar-arterial gradient $\left(\mathrm{P}_{\mathrm{AO}_{2}}-\mathrm{P}_{\mathrm{aO}_{2}}\right), \mathrm{P}_{\mathrm{aO}_{2}} / \mathrm{F}_{\mathrm{IO}_{2}}$, and the oxygenation index (OI): $\left(\overline{\mathrm{P}}_{\mathrm{aw}} \times \mathrm{F}_{\mathrm{IO}_{2}}\right) / \mathrm{P}_{\mathrm{aO}} \cdot{ }^{5}$ Currently, there is no universal consensus among pediatric intensivists of specific values for these calculations that indicate or contraindicate placement of a patient on rescue therapy, such as ECMO. The Pediatric Acute Lung Injury Consensus Conference (PALICC) has defined pediatric ARDS, which builds on the Berlin Definition of adult ARDS in adults. ${ }^{6}$ As part of this definition, OI is recognized as the primary indicator for respiratory disease severity stratification in mechanically ventilated patients. ${ }^{6}$ Historically, an OI of $>40$ or a sustained OI between 30 and 40 was associated with high mortality and used as a criterion for consideration of ECMO in infants and children. However, more recent data suggest that mortality risk may significantly increase at lower levels of OI. ${ }^{7}$

Survival of children with respiratory failure also varies across underlying disorders, diagnoses, and co-morbid conditions. OI has been linked to both duration of mechanical ventilation and mortality.,5,89 A previous study of 131 children with respiratory failure found maximum OI to be an independent predictor of mortality but could not identify a specific OI threshold for risk of death. ${ }^{5}$ Another small study of pediatric stem cell transplant recipients with respiratory failure found that an OI $>20$ was associated with $94 \%$ mortality, whereas subjects with an OI $>25$ had $100 \%$ mortality. ${ }^{9}$ More recently, the PALICC OI-based severity stratification categories of pediatric ARDS were validated to indicate the highest risk of death in the severe category with $\mathrm{OI}>16 .{ }^{10}$

The objectives of this study are to independently characterize retrospective OI data between survivors and nonsurvivors using a single-center experience, with the aim of adding to ongoing efforts to validate PALICC's OI-based pediatric ARDS definition. Additionally, our study examines the discriminatory ability of maximum OI over mean OI while validating PALICC's OI-based pediatric ARDS severity stratification. Finally, this study examines the utility of the electronic medical record as an ongoing source of data for providing serial calculations of the OI.

\section{QUICK LOOK}

\section{Current knowledge}

The oxygenation index $(\mathrm{OI})$ of a patient has been linked to both duration of mechanical ventilation and mortality, with previous studies establishing maximum OI as an independent predictor of mortality. The Pediatric Acute Lung Injury Consensus Conference has defined pediatric ARDS and has recognized OI as the primary matrix for respiratory disease severity stratification in mechanically ventilated patients. However, no specific OI value has been identified that increases the risk of death.

\section{What this paper contributes to our knowledge}

This study investigated the association between OI and mortality, and found that lower OI thresholds than currently used may be associated with adverse outcomes. Odds ratios indicated a 2.4-fold increase in the odds of death $(P=.09,95 \%$ CI $0.9-6.6)$ for each increasing point in maximum OI and superior discriminatory ability of maximum OI over mean OI. The death rate with OI $>17$ was $18 \%$, compared with 6-7\% with OI $<17$. We also evaluated the electronic medical record's current capability to serially calculate a specific respiratory severity index.

\section{Methods}

We conducted a retrospective, single-center study with data collected from electronic medical records for subjects age 1 month (to exclude neonatal physiology) to $20 \mathrm{y}$, who were mechanically ventilated for $>24 \mathrm{~h}$ in the pediatric ICU of Phoenix Children's Hospital from December 2011 through March 2014. Once these inclusion criteria were met, OI data were collected for the entire duration of mechanical ventilation. Survivors were defined as those subjects who were discharged from the hospital, whereas nonsurvivors were subjects who died before discharge. Patients with known intracardiac shunts or cyanotic heart disease were excluded from the study, given the expected decrease in $\mathrm{P}_{\mathrm{aO}_{2}}$ and resultant upward skew in OI data. Subjects with chronic lung diseases and other conditions were included, given variable effects of disease severity on OI. The Phoenix Children's Hospital institutional review board reviewed and approved the study. Pediatric ICU caregivers received no detailed information before the study implementation and were not aware that the electronic medical record was being used to obtain data.

General patient management of progressive respiratory failure in the pediatric ICU at Phoenix Children's Hospital follows mechanical ventilation protocols, which focus 


\section{OI Threshold Associated With Mortality in Pediatric ARF}

on the use of pressure-limited ventilation with tidal volumes $<6 \mathrm{~mL} / \mathrm{kg}$ and peak pressures of $<30-35 \mathrm{~cm} \mathrm{H}_{2} \mathrm{O}$, use of positive end-expiratory pressures of $>5 \mathrm{~cm} \mathrm{H}_{2} \mathrm{O}$, and limitation of inspired oxygen to $<60 \%$ to maintain saturations of $>90 \%$. Use of lower oxygen saturations is permitted in severe cases if hemodynamics and tissue oxygen delivery appear adequate. Use of other modalities, such as high-frequency ventilation, inhaled nitric oxide, surfactant, and ECMO was at the clinician's discretion, and no formal treatment algorithm was employed. No preceding power analysis or further sub-classification of subjects with regard to subsequent rescue therapy beyond mechanical ventilation was examined in this study.

Although 148 subjects met study criteria and were included in the initial study population, complete data for serial $\overline{\mathrm{P}}_{\mathrm{aw}}, \mathrm{F}_{\mathrm{IO}_{2}}$, and $\mathrm{P}_{\mathrm{aO}}$ were extractable from the electronic medical record (AllScripts, Chicago, Illinois) for only 65 subjects (44\%). To obtain $\mathrm{P}_{\mathrm{aO}}$ values that were relatively concurrent with $\overline{\mathrm{P}}_{\mathrm{aw}}$ and $\mathrm{F}_{\mathrm{IO}_{2}}$ data, measurements were matched within concurrent $4 \mathrm{~h}$ time periods surrounding arterial blood gas analyses. A $4 \mathrm{~h}$ window was chosen because this was the likely interval within which nursing staff at Phoenix Children's Hospital would manually $\log$ variables. The number of OIs obtained per subject varied with the frequency of arterial blood gas collection as dictated by clinical course. Serial, mean, and maximum OI were calculated over time for each subject, as well as the medians and interquartile ranges (IQRs). Age of subjects, length of hospital stay, duration of mechanical ventilation, and outcome were also assessed.

Data were analyzed to characterize and compare the mean and maximum OI for all subjects and between the survivor and non-survivor groups. The data were not normally distributed and were therefore presented as the median and interquartile range. Counts and percentages of subjects determined the quartiles, which were used as guides in establishing cut-points for risk of death. Comparisons of continuous data employed the non-parametric Wilcoxon rank-sum test. Receiver operating characteristic analysis was used to assess the discriminant ability of mean and maximum OI for survival. To estimate the association between mean and maximum OI with the likelihood of survival, the data were log-transformed and analyzed with logistic regression.

Logistic regression was used to obtain odds ratios (ORs) for risk of death per $1 \%$ increase in both maximum and mean OIs by determining the exponential function of the regression coefficient. Logistic regression was also implemented to determine the potential confounding bias of age, hospital length of stay, and the days receiving mechanical ventilation. Covariates that changed the original point estimate by $>10 \%{ }^{11}$ were identified as causing potential confounding bias, leading to the addition of patient age and days of mechanical ventilation to the final model. The OR and $95 \% \mathrm{CI}$ are presented, which indicated the likelihood of non-survival for $1 \%$ increments in maximum OI and mean OI. Statistical significance was defined as an $\alpha$ of .05 , with 2 -sided alternative hypotheses. Analyses were conducted using STATA 13 (StataCorp, College Station, Texas).

\section{Results}

Serial OIs were calculated on 65 subjects from electronic medical record data. Fifty-nine subjects (91\%) survived hospitalization (survivors) and were discharged, whereas 6 died (non-survivors) (9\%). Both the survivor and non-survivor groups were demographically representative of the larger patient population of Phoenix Children's Hospital. Pulmonary pathology represented $31 \%$ of all primary diagnoses in the survivor group and $17 \%$ in the non-survivor group. A wide variety of secondary diagnoses were represented. However, $81 \%$ of survivors and $67 \%$ of non-survivors carried a secondary diagnosis of acute respiratory failure. $14 \%$ of the survivors and $17 \%$ of the non-survivor group endorsed chronic pulmonary conditions (Table 1).

In addition to pressure-limited ventilation, 14 subjects received other advanced respiratory therapies, which included 4 receiving intrapulmonary percussive ventilation, 4 receiving inhaled nitric oxide, 4 receiving high-frequency oscillation ventilation, and 2 receiving adaptive servo ventilation. Of these, 2 subjects were non-survivors: 1 received both inhaled nitric oxide and high-frequency oscillation ventilation, and another received intrapulmonary percussive ventilation. In the survivor group, 3 subjects received intrapulmonary percussive ventilation, 3 received inhaled nitric oxide, and 3 received high-frequency oscillation ventilation.

The number of OIs calculable for each subject varied significantly given the parameters for inclusion of variables. The median number of OIs was 11 for survivors with IQR of $26-4$, and 18 for non-survivors with IQR of 24-12. The median maximum OI was 10 for all subjects, with IQR of $17-4,8$ for survivors with an IQR of 17-4, and 17 for nonsurvivors with an IQR of $51-9(P=.14$ via Wilcoxon ranksum test) (Fig. 1). The median mean OI was 5 for all subjects with an IQR of 10-2, 4 for survivors with an IQR of 10-2, and 6 for non-survivors with an IQR of 9-3 $(P=.48)$.

Receiver operating characteristic analysis of maximum and mean OI demonstrated an advantage of maximum OI over mean OI in discriminatory ability. The area under the curve for maximum OI was 0.68 , whereas mean OI area under the curve was 0.58 (Fig. 2).

Cut-points for risk of death were examined after establishing count-determined quartiles. The number of nonsurvivor and survivor subjects within each quartile were counted. Analysis within each quartile indicated that mor- 


\section{OI Threshold Associated With Mortality in Pediatric ARF}

Table 1. Subject Demographics: Primary Diagnoses, Most Common Secondary Diagnoses, and Chronic Conditions of Survivor and Non-Survivor Groups

\begin{tabular}{|c|c|c|}
\hline Primary Diagnoses $(n)$ & Secondary Diagnoses $(n)$ & Chronic Conditions $(n)$ \\
\hline \multicolumn{3}{|l|}{ Survivors } \\
\hline $\begin{array}{l}\text { Brain hemorrhage and TBI (child } \\
\text { physical abuse, skull base fractures, } \\
\text { other trauma) (8) }\end{array}$ & Acute respiratory failure (48) & $\begin{array}{l}\text { Musculoskeletal pathologies (including spine, hip, } \\
\text { caudal regression, paralysis) (24) }\end{array}$ \\
\hline Musculoskeletal postoperative (8) & $\begin{array}{l}\text { Brain injury (TBI, ischemia, hemorrhage, } \\
\text { epilepsy, coma) (41) }\end{array}$ & Encephalopathies (20) \\
\hline $\begin{array}{l}\text { Acute bronchiolitis due to respiratory } \\
\text { syncytial virus ( } 7 \text { ) }\end{array}$ & GI (feeding problems, dysphagia, FTT) (37) & $\begin{array}{l}\text { Chronic lung disease/pulmonary pathologies } \\
\text { (bronchopulmonary dysplasia, trachoemalacia, } \\
\text { pneumatocele, sleep apnea, fibrosis, asthma) (18) }\end{array}$ \\
\hline $\begin{array}{l}\text { Other surgical postoperative (volvulus, } \\
\text { omphalocele, renal transplant ( } 7 \text { ) }\end{array}$ & Infection (29) & GI (gastrostomy tube-dependent, dysphagia) (19) \\
\hline Acute respiratory failure (6) & Other pulmonary pathologies (26) & $\begin{array}{l}\text { Chronic heart disease (congenital, dysrhythmia, } \\
\text { murmur) (14) }\end{array}$ \\
\hline Pneumonia (5) & $\begin{array}{l}\text { Cardiac abnormalities (arrythmias, arrest, } \\
\text { cardiogenic shock, HTN, hypotension) (20) }\end{array}$ & Seizures/epilepsy (13) \\
\hline Brain malignancy (5) & Anemia $(22)$ & Developmental delay (10) \\
\hline Septicemia (3) & Pneumonia (20) & Chromosomal abnormalities (9) \\
\hline Refractory seizures (2) & Hypoxemia/acidosis (17) & Endocrine disorders (panhypopit, hypothyroidism) (9) \\
\hline Hypoosmolality/hyperosmolality (2) & Acute tracheitis/bronchiolitis (17) & $\begin{array}{l}\text { Other congenital (traceheoesophageal fistula, } \\
\text { laryngeal cleft, omphalocele, choledochal cyst, } \\
\text { renal agenesis) ( } 7 \text { ) }\end{array}$ \\
\hline Meningitis (2) & Sepsis/SIRS (15) & Ventilator status (4) \\
\hline Hydrocephalus (2) & $\begin{array}{l}\text { Muscular pathology (hypotonia, paralysis, } \\
\text { weakness) (11) }\end{array}$ & $\begin{array}{l}\text { Malignant and benign neoplasm (melanoma, brain, } \\
\text { hemangioma) (4) }\end{array}$ \\
\hline Bordetella pertussis (2) & Coagulation defect/abnormality (8) & Blood disorders (Factor V Leidan, anemia) (3) \\
\hline Cardiac arrest/failure (1) & Acute kidney injury and failure (8) & Inadequate physical development/failure to thrive (2) \\
\hline \multicolumn{3}{|l|}{ Non-survivors } \\
\hline TBI (skull base fracture) (2) & $\begin{array}{l}\text { Brain injury (TBI, ischemia, hemorrhage, } \\
\text { epilepsy, coma) (6) }\end{array}$ & Chromosomal abnormalities (2) \\
\hline Septicemia (2) & Acute respiratory failure (4) & $\begin{array}{l}\text { Chronic heart disease (congenital, dysrhythmia, } \\
\text { murmur) (1) }\end{array}$ \\
\hline Pneumonia (1) & $\begin{array}{l}\text { Other pulmonary pathologies (emphysema, } \\
\text { pneumothorax, pneumonitis, pulmonary HTN, } \\
\text { restrictive lung disease, empyema, effusion, } \\
\text { bronchiectasis, apnea, esophagitis) (3) }\end{array}$ & $\begin{array}{l}\text { Chronic lung disease/pulmonary pathologies } \\
\text { (bronchopulmonary dysplasia, trachoemalacia, } \\
\text { pneumatocele, sleep apnea, fibrosis) (1) }\end{array}$ \\
\hline Cardiac arrest, unknown cause (1) & $\begin{array}{l}\text { Cardiac abnormalities (arrythmias, arrest, } \\
\text { cardiogenic shock, HTN, hypotension) (3) }\end{array}$ & $\begin{array}{l}\text { Skeletal pathologies (including spine, hip, caudal } \\
\text { regression) (1) }\end{array}$ \\
\hline & Coagulation defect/abnormality (2) & Quadriplegia, diplegia (1) \\
\hline $\begin{array}{l}\text { TBI }=\text { traumatic brain injury } \\
\text { GI }=\text { gastrointestinal } \\
\text { FTT = failure to thrive } \\
\text { HTN }=\text { hypertension } \\
\text { SIRS }=\text { systemic inflammatory response syndrome }\end{array}$ & & \\
\hline
\end{tabular}

tality was unchanged $(6-7 \%)$ until maximum OI $>17$. Mortality increased to $18 \%$ with OI $>17$.

The median age for all subjects was $2.8 \mathrm{y}$ with IQR of 12-0.7 $\mathrm{y}$. The median age for survivors was $2.4 \mathrm{y}$ with an IQR of 12-0.5 y, and the median age was $10.4 \mathrm{y}$ for non-survivors with an IQR of $14-5$ y $(P=.12)$. Length of hospital stay was statistically significant, with median length of stay of $18 d$ for all subjects with an IQR of 31-9 $\mathrm{d}, 21 \mathrm{~d}$ for survivors with an IQR of 36-10 d, and $4 \mathrm{~d}$ for non-survivors with an IQR of 6-3 d. Duration of mechanical ventilation for subjects was measured from first recorded $\overline{\mathrm{P}}_{\mathrm{aw}}$ to last recorded $\overline{\mathrm{P}}_{\mathrm{aw}}$. The median duration of mechanical ventilation for all subjects was $6.5 \mathrm{~d}$ with an IQR of 11-3 d, $7 \mathrm{~d}$ for survivors with an IQR of 12-3 d, and $3 \mathrm{~d}$ for non-survivors with an IQR of 5-2 d $(P=.21)$.

After adjusting for age and days of mechanical ventilation, an OR of 2.4 was determined for odds of death with each $1 \%$ increase in maximum OI $(P=.09)$ over the 


\section{OI Threshold Associated With Mortality in Pediatric ARF}
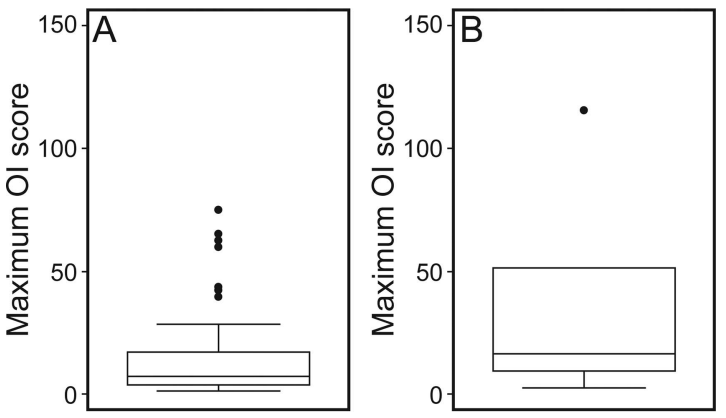

Fig. 1. Box plots of maximum oxygenation index (OI) in survivors (A) and non-survivors (B), which demonstrate a higher median maximum $\mathrm{Ol}$ as well as increased dispersion from the mean for the non-survivor cohort. Boxes represent 75th and 25th percentiles, center lines denote the median, whiskers show 90th and 10th percentiles, and points show outliers.

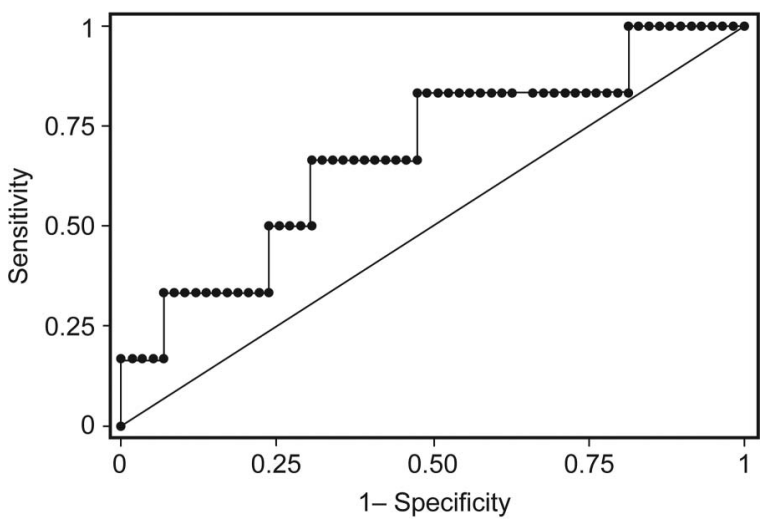

Fig. 2. Receiver operating characteristic analysis of maximum oxygenation index versus risk of death with area under the curve $=$ 0.68 , indicative of increased discriminatory performance.

course of hospitalization, with $95 \%$ CI of $0.9-6.6$. Mean OI resulted in an OR of 1.9, thus increasing the odds of death for each $1 \%$ increase in mean OI $(P=.25)$ over the length of hospitalization, with $95 \%$ CI $0.6-5.9$.

\section{Discussion}

In this study, the mortality for subjects with OIs $>17$ was $18 \%$. Although this small study does not stand alone in providing definitive results, the implications of the identified trends are clinically relevant. Although these results may seem modest, it should be noted that overall mortality in the pediatric ICU at our institution (and generally, in pediatric ICUs nationwide) is $2-3 \% .^{12}$ Thus, this mortality in subjects with respiratory failure is substantially higher than baseline mortality rates and represents a high-risk group. Historically, an OI of $>40$ in infants was noted to be associated with high mortality and was used to identify candidates for rescue therapies, such as ECMO. The current report validates other single-center studies and the international PALICC group guidance that lower value OIs may be associated with adverse outcome. ${ }^{6,7,10}$ Our findings of superior discriminatory ability and increased OR of maximum OI over mean OI, while validating PALICC's OI-based pediatric ARDS severity stratification, lend support to pediatric ARDS physiologic response theories that emphasize data analysis and severity stratification following $24 \mathrm{~h}$ of initial post-injury stabilization and resuscitation efforts. This approach more accurately reflects the true degree of lung injury, as it allows for improvement in ventilation/perfusion mismatches, excludes $\mathrm{P}_{\mathrm{aO}}$ data obtained before complete resuscitation, and incorporates increased lung recruitment. ${ }^{10}$ Quantifying the predictive capability of the OI and identifying a threshold value for escalation of therapy will help guide discussion regarding the appropriate time of rescue intervention. In addition, the use of lower OIs for entry criteria into clinical studies of pediatric respiratory failure may also be warranted. $6,10,13-15$ This threshold OI finding warrants further investigation with larger data sets from multiple centers.

In our study, review of the subject electronic medical record had limited capability in calculating OI in that recorded $\mathrm{P}_{\mathrm{aO}}$ values were often infrequent and outside the $4 \mathrm{~h}$ window of associated $\overline{\mathrm{P}}_{\mathrm{aw}}$ values and $\mathrm{F}_{\mathrm{IO}_{2}}$. Only $44 \%$ of subjects had the necessary data recorded within the specified time frame to calculate OI. To improve data capture rates in the future, pediatric ICU personnel worked with electronic medical record programmers to define more specific data entry times and alerts when specific parameters (those involved in calculation of oxygenation index in this case) are met. With continued refinements, possible inclusion of real-time OI calculations in an electronic medical record data-derived severity score algorithm that triggers pre-established therapy escalation alerts has the potential to improve the timeliness of interventions that may improve outcome.

The major limitation of our study was a small sample size after establishing a $4 \mathrm{~h}$ window in which $\mathrm{P}_{\mathrm{aO}}$ values were recorded in association with $\overline{\mathrm{P}}_{\mathrm{aw}}$ values and $\mathrm{F}_{\mathrm{IO}_{2}}$. Prior power analysis to determine numbers of subjects for statistically significant results as well as more diligent recording of OI variables in relation to ventilator settings would be beneficial for future studies. Further consideration of the $4 \mathrm{~h}$ window is also warranted. Although we feel that it is a reasonable time frame for including variables representative of the clinical scenario, we acknowledge that much can change during this interval, making this a challenging limitation for this retrospective study. Additionally, future comprehensive data studies may find less inherent bias toward sicker subjects, who probably have more frequent OI measurements, in comparing data during a fixed time frame rather than the entire duration of mechanical ventilation. 


\section{OI Threshold Associated With Mortality in Pediatric ARF}

Of note, because patients with respiratory failure may not have an indwelling arterial line from which $\mathrm{P}_{\mathrm{aO}_{2}}$ can be determined, the PALICC recommendations for pediatric ARDS include an oxygenation saturation index as an indicator of severity. In this equation, the $\mathrm{P}_{\mathrm{aO}_{2}}$ is replaced by the arterial oxygen saturation. This score was not calculated in this study, because this recommendation appeared after the study analysis was completed.

\section{Conclusions}

We found a nearly 3 -fold increase in the risk of mortality with each increasing point in maximum OI, and an OI threshold of 17 that triples the risk of death. The superior discriminatory ability of maximum over mean OI also implies that previous studies are correct to refute the use of OI data in the first $24 \mathrm{~h}$ of mechanical ventilation as representative of the degree of lung injury and predictive of outcome. ${ }^{10}$ Establishing reproducible critical OI thresholds for risk of mortality may permit the creation of alert algorithms for the escalation of support, whether advanced respiratory or extracorporeal, in the current era. To this end, our work supports lower thresholds of OI for defining respiratory disease severity as outlined in the newly established pediatric ARDS definition. Historically, entry criteria for rescue therapies, such as ECMO, generally included significantly higher OI values. Given the increase in mortality at a value much lower than previously suggested, our work supports the need for significantly larger, multicenter studies to examine similar trends and determine statistically significant predictive OI values, with the potential to lower OI application thresholds of rescue therapies. The ubiquity of the electronic medical record is presenting greater possibilities for ease of data access, reconciliation, and functional presentation. Although retrospective application of this technology has limitations, as we have shown, the potential is there to offer real-time support in selecting the best treatment modalities in critically ill patients. Electronic medical record clinical decision tools to track the progression of acute respiratory distress in the pediatric ICU for escalation of rescue therapies, which includes serial and maximum OI calculation, appear feasible and may prove valuable in the future.

\section{REFERENCES}

1. Monchi M, Bellenfant F, Cariou A, Joly LM, Thebert D, Laurent I, et al. Early predictive factors of survival in the acute respiratory distress syndrome: a multivariate analysis. Am J Respir Crit Care Med 1998;158(4):1076-1081.

2. Pathan N, Ridout DA, Smith E, Goldman AP, Brown KL. Predictors of outcome for children requiring respiratory extra-corporeal life support: implications for inclusion and exclusion criteria. Intensive Care Med 2008;34(12):2256-2263.

3. Quasney MW, López-Fernández YM, Santschi M, Watson RS, Pediatric Acute Lung Injury Consensus Conference Group). Pediatric Acute Lung Injury Consensus Conference Group. The outcomes of children with pediatric acute respiratory distress syndrome: proceedings from the Pediatric Acute Lung Injury Consensus Conference. Pediatr Crit Care Med 2015;16(5 Suppl 1):S118-S131.

4. Dalton HJ, Butt WW. Extracorporeal life support: an update of Rogers' Textbook of Pediatric Intensive Care. Pediatr Crit Care Med 2012;13(4):461-471.

5. Trachsel D, McCrindle BW, Nakagawa S, Bohn D. Oxygenation index predicts outcome in children with acute hypoxemic respiratory failure. Am J Respir Crit Care Med 2005;172(2):206-211.

6. Khemani RG, Smith LS, Zimmerman JJ, Erickson S, Pediatric Acute Lung Injury Consensus Conference Group. Pediatric acute respiratory distress syndrome: definition, incidence, and epidemiology: proceedings from the Pediatric Acute Lung Injury Consensus Conference. Pediatr Crit Care Med 2015;16(5):S23-S40.

7. Ghuman AK, Newth CJ, Khemani RG. The association between the end tidal alveolar dead space fraction and mortality in pediatric acute hypoxemic respiratory failure. Pediatr Crit Care Med 2012;13(1):1115.

8. Karimova A, Brown K, Ridout D, Beierlein W, Cassidy J, Smith J, et al. Neonatal extracorporeal membrane oxygenation: practice patterns and predictors of outcome in the UK. Arch Dis Child Fetal Neonatal Ed 2009;94(2):F129-F132.

9. Rowan CM, Hege KM, Speicher RH, Goodman M, Perkins SM, Slaven JE, et al. Oxygenation index predicts mortality in pediatric stem cell transplant recipients requiring mechanical ventilation. Pediatr Transplant 2012;16(6):645-650.

10. Yehya N, Servaes S, Thomas NJ. Characterizing degree of lung injury in pediatric acute respiratory distress syndrome. Crit Care Med 2015;43(5):937-946.

11. Mickey RM, Greenland S. The impact of confounder selection criteria on effect estimation. Am J Epidemiol 1989;129(1):125-137.

12. Markovitz BP, Kukuyeva I, Soto-Campos G, Khemani RG. PICU volume and outcome: a severity-adjusted analysis. Pediatr Crit Care Med 2016;17(6):483-489.

13. Khemani RG, Thomas NJ, Venketachalam V, Scimeme JP, Berutti $\mathrm{T}$, Schneider JB, et al. Comparison of $\mathrm{S}_{\mathrm{pO}_{2}}$ to $\mathrm{P}_{\mathrm{aO}}$ based markers of lung disease severity for children with acute lung injury. Crit Care Med 2012;40(4):1309-1316.

14. Bermudez CA, Rocha RV, Zaldonis D, Bhama JK, Crespo MM, Shigemura N, et al. Extracorporeal membrane oxygenation as a bridge to lung transplant: midterm outcomes. Ann Thorac Surg 2011;92(4): 1226-1231; discussion 1231-1232.

15. Fiser SM, Kron IL, McLendon Long S, Kaza AK, Kern JA, Tribble CG. Early intervention after severe oxygenation index elevation improves survival following lung transplant. J Heart Lung Transplant 2001;20(6):631-636.

This article is approved for Continuing Respiratory Care Education credit. For information and to obtain your CRCE

(free to AARC members) visit www.rcjournal.com

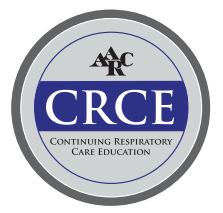

